Arab Univ. J. Agric. Sci., Ain Shams Univ., Cairo, 14(1), 195-203, 2006

\title{
EFFECT OF NITROGENOUS AND PHOSPHATIC FERTILIZERS ON PERFORMANCE OF RAINFED PEARL MILLET (PENNISETUM GLAUCUM L.) GROWN ON CLAY LOAM SOIL
}

\author{
Eltilib $^{1}$, A.M.A.; T.E.M. Hago ${ }^{1}$; A.H. Awad Elkarim ${ }^{1, a}$ \\ and S.A.M. Ali ${ }^{2}$
}

\begin{abstract}
An experiment was conducted for two summer seasons (2000 and 2001) to study the effect of nitrogenous and phosphatic fertilizers on performance of rainfed pearl millet (Pennisetum glaucum L.) grown on clay loam soil at University of Zalingei site, Western Darfour states, Sudan. The experimental design used was spilt-plot design with five replications. Four nitrogen treatments $(0,30,60$ and $80 \mathrm{~kg} \mathrm{~N} / \mathrm{ha})$ and four phosphorus treatments $(0,15,30$ and $60 \mathrm{~kg}$ P/ha) were used. Nitrogen treatments were assigned to the main plots and phosphorus levels were allotted to the sub-plots. Urea $(46 \% \mathrm{~N})$ and triple superphosphate $\left(48 \% \mathrm{P}_{2} \mathrm{O}_{5}\right)$ were used as sources of nitrogen and phosphorus, respectively. Phosphorus treatments were applied at sowing, whereas nitrogen fertilizer was split into two equal doses, one was added four days after emergence and the second was three weeks after sowing. Results of the experiments showed that application of nitrogen increased the plant height over the control. The increments were in range of $5.7 \%-16.1 \%$. Leaf area index (LAI), grain and stover yields were significantly affected by nitrogen application. The increments over control were in the range of $39 \%-92.3 \%, 10.1 \%-112.7 \%$ and $34.1 \%$ - $126.9 \%$ for LAI, grain yield and stover yield, respectively. Regarding phosphorus, the results revealed that phosphorus addition had a significant effect on plant height, LAI, grain and stover yields. The increments over control ranged from $2.4 \%$ $11.3 \%, 6.3 \%-17.9 \%, 6.7 \%-24.6 \%$ and $6.8 \%-35.4 \%$ for plant height, LAI, grain and stover yields, respectively. The interaction between nitrogen and phosphorus had a significant effect only on LAI and plant height at the sixth week measurement in the first and second seasons, respectively. The tallest plants were obtained from $3 \mathrm{~N} \times 3 \mathrm{P}$ treatment whereas the greatest LAI was obtained by $3 \mathrm{~N} \times 2 \mathrm{P}$ treatment.
\end{abstract}

Keywords: Phosphatic fertilizers, Pearl millet, Plant height, Leaf area index, Grain yield

1- Department of Soils \& Environment Sciences, Faculty of Agriculture, University of Khartoum, Shambat 13314, Sudan

2- Faculty of Agriculture, University of Zalingie, Western Darfour State, Sudan a- E-mail: akarim61@yahoo.com

(Received September 23, 2005) (Accepted December 12, 2005) 


\section{INTRODUCTION}

Soils, particularly in the arid and semiarid regions, rarely contain adequate amounts of essential plant nutrients to sustain high yields of most crops. This is particularly true for nitrogen and mostly for phosphorus. Use of chemical fertilizers has been practiced for a long time in modern agriculture to compensate these deficiencies in nitrogen and phosphorus.

In Sudan, pearl millet is an important cereal crop, next to sorghum. It constitutes the stable food of the majority of Western Sudan (Darfur and Kordofan) where it occupies an area of $1.2-2.9$ million hectares. Most of the area is cultivated and harvested under traditional rainfed agriculture (Abuelgasim and Jain, 1987; FAO, 1999). It is also cultivated on alluvial soil along wadis. Recently, some farmers in the mechanized clay plain of central Sudan started to grow pearl millet instead of sorghum which is susceptible to Striga hermonthica (Elmahi, et al 1995). Very little research work was conducted in this sector compared with irrigated agriculture. In particular, pearl millet did not receive the appropriate attention. Therefore, the present study was conducted to investigate the effect of nitrogen and phosphorus fertilization on performance and yield of pearl millet grown on clay loam soil under Western Sudan conditions.

\section{MATERIAL AND METHODS}

An experiment was carried out for two summer seasons (2000 and 2001) to investigate the effect of nitrogen and phosphorus fertilizers on growth and yield of rainfed pearl millet (Pennisetum glaucum). The study site was at the Uni- versity of Zalingei, Western Darfur state (latitude $12^{\circ} 45^{\prime} \mathrm{N}$ and longitude $23^{\circ} 29^{\prime}$ $\mathrm{E}$ with an attitude of $900 \mathrm{~m}$ above mean sea level).

The soil of the site is clay loam $(41 \%$ clay), $\mathrm{pH}$ range from 6.4 to 7.0 with low available soil phosphorus $(0.83-0.9$ ppm). According to Black et al (1969) standards, the soil organic carbon is low $(0.25-0.32 \%)$ and, hence, low soil nitrogen content $(0.029-0.035 \%)$. The experimental design used was spilt-plot design replicated five times with nitrogen in the main plots and phosphorus in the subplots. The area of main plots and subplots was $108 \mathrm{~m}^{2}$ and $27 \mathrm{~m}^{2}$, respectively. A local pearl millet cultivar (Darmasa) was sown on the $12^{\text {th }}$ and $15^{\text {th }}$ of July in the first and second seasons, respectively. Traditional hand sowing was used by using local implement. Nitrogen treatments consisted of four $\mathrm{N}$ levels: 0,30 , 60 and $80 \mathrm{~kg} \mathrm{~N} /$ ha designated as $0 \mathrm{~N}, 1 \mathrm{~N}$, $2 \mathrm{~N}$ and $3 \mathrm{~N}$, respectively, phosphorus treatments consisted of four P levels: 0 , 15,30 and $60 \mathrm{~kg} \mathrm{P} / \mathrm{ha}$ designed as $0 \mathrm{P}, 1 \mathrm{P}$, $2 \mathrm{P}$ and $3 \mathrm{P}$, respectively. Urea $(46 \% \mathrm{~N})$ and triplesuperphosphate $\left(\begin{array}{lll}48 \% & \mathrm{P}_{2} \mathrm{O}_{5}\end{array}\right)$ were used as sources of nitrogen and phosphorus, respectively. Triplesuperphosphate was applied at sowing in bands of $5 \mathrm{~cm}$ depth and at a distance of $5 \mathrm{~cm}$ from the seeds. Urea was split into equal doses, one dose added four days after germination and the other was three weeks after sowing by hand placement method. Thinning was done when the plant were four weeks old to end up with three plants per hole. Growth parameters, plant height and leaf area index (LAI) were measured at an interval of two weeks starting a month after sowing until harvest. The crop was harvested at 120 and 112 days from sowing in the first and 
second seasons, respectively. Grain and stover yields were determined in an area of $6.25 \mathrm{~m}^{2}$ in the centre of the middle three rows in each plot.

\section{RESULTS AND DISCUSSION}

Nitrogen application had a significant effect $(\mathrm{P} \leq 0.05)$ on plant height (Table, 1). The plant height was significantly increased over the control by nitrogen treatment. The increases in plant height produced by $1 \mathrm{~N}, 2 \mathrm{~N}$ and $3 \mathrm{~N}$ treatments over the control at $12^{\text {th }}$ week after sowing were $7.1 \%, 10.5 \%, 9.3 \%$ and $5.7 \%$, $11.0 \%$ and $16.1 \%$ for the first and second seasons, respectively. These findings were in conformity with results obtained by Ibrahim, (1996).

Nitrogen application had a significant effect on LAI in both seasons (Table, 2) where LAI index increased with increasing nitrogen rate up to the $8^{\text {th }}$ week of the sowing but this disappeared as the season proceeded. The increments in LAI over the control due to nitrogen application at the rate of $1 \mathrm{~N}, 2 \mathrm{~N}$ and $3 \mathrm{~N}$ at the $8^{\text {th }}$ week measurement were $39.0 \%, 52.4 \%$ and $60.0 \%$, respectively, in the first season and $39.6 \%, 78.6 \%$ and $92.3 \%$, respectively, in the second season. Mourad et al (1986) found that LAI values were significantly increased with increasing nitrogen rate up to $90 \mathrm{~kg} \mathrm{~N} / \mathrm{ha}$, which was almost equivalent to the rate used in this study.

Phosphorus application had a significant effect on plant height (Table, 3). The increments in plant height produced by $\mathrm{P}$ treatments ranged, on average, between $2.4 \%-10.0 \%$ over the control. Similar findings were reported by Okalebo (1984). The added phosphorus increased LAI significantly over the control (Table,
4) for the first three measurements but later this disappeared. These results were in line with those obtained by Elmedani, (1997) who quoted similar results.

Grain and stover yields were significantly affected by nitrogen and phosphorus application (Table, 5). The increments due to nitrogen fertilization were in the range of $10.1 \%-112.7 \%$ and $34.1 \%$ $126.9 \%$ of grain and stover yields, respectively, whereas, the respective increments in grains and stover yields due to phosphorus application ranged between $6.7 \%-24.6 \%$ and $6.8 \%-35.8 \%$.

These results were in line with those obtained by many research workers (Mason, 1989 and Singh \& Sharma, 1995).

The yield of the first season was higher than that of the second season. This was mainly due to the fluctuating rainfall which is a critical factor that determines yield in rainfed agriculture. The rainfall in the first season was favorable for crop growth in terms of total amount (777.8 $\mathrm{mm}$ ) and distribution throughout the growth period whereas in the second season the rainfall was low (421 $\mathrm{mm})$, less than the twenty years mean $(488.4 \mathrm{~mm})$. These results were similar to those reported by Bationo et al (1989) who reported that low rainfall can adversely affect crop productivity.

However, the interaction of $\mathrm{N} \times \mathrm{P}$ had exerted a non significant effect on most measured parameters. Exception of this, LAI at the $6^{\text {th }}$ week after sowing in the first season was significantly affected by treatment interaction (Table, 6). Similarly, plant height recorded at the $6^{\text {th }}$ week measurement in the second season was significantly affected by $\mathrm{N} \times \mathrm{P}$ interaction (Table, 7). 
Table 1. Effect of nitrogen fertilizer on plant height $(\mathrm{cm})$

\begin{tabular}{|cccccc|}
\hline \multirow{2}{*}{ Nitrogen level } & \multicolumn{5}{c|}{ Time (weeks after sowing) } \\
\cline { 2 - 6 } & 4 & 6 & 8 & 10 & 12 \\
\hline \multicolumn{6}{c|}{ First season } \\
\hline $0 \mathrm{~N}$ & $43.56 \mathrm{~b}$ & $96.97 \mathrm{c}$ & $159.48 \mathrm{c}$ & $198.15 \mathrm{~b}$ & $199.98 \mathrm{~b}$ \\
$1 \mathrm{~N}$ & $48.45 \mathrm{ab}$ & $123.70 \mathrm{bc}$ & $189.72 \mathrm{~b}$ & $212.78 \mathrm{a}$ & $214.22 \mathrm{a}$ \\
$2 \mathrm{~N}$ & $53.38 \mathrm{a}$ & $141.24 \mathrm{a}$ & $203.67 \mathrm{a}$ & $220.03 \mathrm{a}$ & $220.89 \mathrm{a}$ \\
$3 \mathrm{~N}$ & $51.61 \mathrm{a}$ & $133.09 \mathrm{~b}$ & $200.70 \mathrm{ab}$ & $218.33 \mathrm{a}$ & $218.51 \mathrm{a}$ \\
\hline \multicolumn{5}{c}{ Second season } \\
\hline $\mathrm{N}$ & $40.80 \mathrm{c}$ & $73.87 \mathrm{~d}$ & $110.28 \mathrm{c}$ & $159.89 \mathrm{c}$ & $161.11 \mathrm{c}$ \\
$2 \mathrm{~N}$ & $45.91 \mathrm{~b}$ & $97.06 \mathrm{c}$ & $133.58 \mathrm{~b}$ & $169.84 \mathrm{bc}$ & $170.23 \mathrm{bc}$ \\
$3 \mathrm{~N}$ & $50.72 \mathrm{a}$ & $105.71 \mathrm{~b}$ & $144.58 \mathrm{~b}$ & $178.57 \mathrm{ab}$ & $178.80 \mathrm{ab}$ \\
& $52.39 \mathrm{a}$ & $116.30 \mathrm{a}$ & $156.23 \mathrm{a}$ & $187.73 \mathrm{a}$ & $187.73 \mathrm{a}$ \\
\hline
\end{tabular}

Means within the same column followed by the same letter are not significantly different at $\mathrm{P} \leq 0.05$ according to Duncan's Multiple Range Test (DMRT).

$0 \mathrm{~N}=0 \mathrm{~kg} \mathrm{~N} / \mathrm{ha} \quad 1 \mathrm{~N}=30 \mathrm{~kg} \mathrm{~N} / \mathrm{ha} \quad 2 \mathrm{~N}=60 \mathrm{~kg} \mathrm{~N} / \mathrm{ha} \quad 3 \mathrm{~N}=80 \mathrm{~kg} \mathrm{~N} / \mathrm{ha}$

Table 2. Effect of nitrogen fertilizer on leaf area index (LAI)

\begin{tabular}{|ccccc|}
\hline \multirow{2}{*}{ Nitrogen level } & \multicolumn{5}{c|}{ Time (weeks after sowing) } \\
\cline { 2 - 5 } & 4 & 6 & 8 & 10 \\
\hline \multicolumn{6}{c}{ First season } \\
\hline $0 \mathrm{~N}$ & $0.76 \mathrm{~b}$ & $2.49 \mathrm{c}$ & $3.13 \mathrm{c}$ & $2.55 \mathrm{a}$ \\
$1 \mathrm{~N}$ & $1.19 \mathrm{a}$ & $3.41 \mathrm{~b}$ & $4.35 \mathrm{~b}$ & $2.65 \mathrm{a}$ \\
$2 \mathrm{~N}$ & $1.35 \mathrm{a}$ & $3.93 \mathrm{a}$ & $4.77 \mathrm{ab}$ & $2.72 \mathrm{a}$ \\
$3 \mathrm{~N}$ & $1.26 \mathrm{a}$ & $3.81 \mathrm{ab}$ & $5.01 \mathrm{a}$ & $2.83 \mathrm{a}$ \\
\hline & Second season & & \\
\hline $0 \mathrm{~N}$ & $0.65 \mathrm{c}$ & $1.09 \mathrm{c}$ & $1.54 \mathrm{c}$ & $1.10 \mathrm{a}$ \\
$1 \mathrm{~N}$ & $0.91 \mathrm{~b}$ & $1.67 \mathrm{~b}$ & $2.15 \mathrm{~b}$ & $1.12 \mathrm{a}$ \\
$2 \mathrm{~N}$ & $1.17 \mathrm{a}$ & $2.14 \mathrm{a}$ & $2.75 \mathrm{a}$ & $1.43 \mathrm{a}$ \\
$3 \mathrm{~N}$ & $1.27 \mathrm{a}$ & $2.29 \mathrm{a}$ & $2.97 \mathrm{a}$ & $1.50 \mathrm{a}$ \\
\hline
\end{tabular}

Means within the same column followed by the same letter are not significantly different at $\mathrm{P} \leq 0.05$ according to Duncan's Multiple Range Test (DMRT).

Symbols as shown in Table 1

Arab Univ. J. Agric. Sci., 14(1), 2006 
Table 3. Effect of phosphorus fertilizer on plant height $(\mathrm{cm})$

\begin{tabular}{|cccccc|}
\hline \multirow{2}{*}{ Phosphorus level } & \multicolumn{5}{c|}{ Time (weeks after sowing) } \\
\cline { 2 - 6 } & 4 & 6 & 8 & 10 & 12 \\
\hline \multicolumn{5}{c|}{ First season } \\
\hline 0 P & $44.68 \mathrm{~b}$ & $111.04 \mathrm{c}$ & $125.90 \mathrm{c}$ & $205.79 \mathrm{c}$ & $207.31 \mathrm{c}$ \\
$1 \mathrm{P}$ & $50.06 \mathrm{a}$ & $124.24 \mathrm{~b}$ & $188.00 \mathrm{bc}$ & $211.34 \mathrm{~b}$ & $212.35 \mathrm{bc}$ \\
$2 \mathrm{P}$ & $51.34 \mathrm{a}$ & $125.59 \mathrm{~b}$ & $197.44 \mathrm{a}$ & $218.70 \mathrm{ab}$ & $219.03 \mathrm{a}$ \\
$3 \mathrm{P}$ & $50.91 \mathrm{a}$ & $134.13 \mathrm{a}$ & $192.25 \mathrm{ab}$ & $213.46 \mathrm{a}$ & $214.93 \mathrm{ab}$ \\
\hline & \multicolumn{5}{c}{ Second season } \\
\hline 1 P & $43.01 \mathrm{c}$ & $83.35 \mathrm{c}$ & $120.28 \mathrm{c}$ & $162.58 \mathrm{c}$ & $163.00 \mathrm{c}$ \\
$2 \mathrm{P}$ & $47.40 \mathrm{~b}$ & $99.77 \mathrm{~b}$ & $136.67 \mathrm{~b}$ & $173.97 \mathrm{~b}$ & $174.08 \mathrm{~b}$ \\
$3 \mathrm{P}$ & $49.34 \mathrm{a}$ & $103.63 \mathrm{ab}$ & $142.29 \mathrm{a}$ & $178.55 \mathrm{ab}$ & $179.36 \mathrm{ab}$ \\
\hline & $50.07 \mathrm{a}$ & $106.19 \mathrm{a}$ & $145.43 \mathrm{a}$ & $180.94 \mathrm{a}$ & $181.43 \mathrm{a}$ \\
\hline
\end{tabular}

Means within the same column followed by the same letter are not significantly different at $\mathrm{P} \leq 0.05$ according to Duncan's Multiple Range Test (DMRT).

$0 \mathrm{P}=0 \mathrm{~kg} \mathrm{P} / \mathrm{ha} \quad 1 \mathrm{P}=15 \mathrm{~kg} \mathrm{P} / \mathrm{ha} \quad 2 \mathrm{P}=30 \mathrm{~kg} \mathrm{P} / \mathrm{ha} \quad 3 \mathrm{P}=60 \mathrm{~kg} \mathrm{P} / \mathrm{ha}$

Table 4. Effect of phosphorus fertilizer on leaf area index (LAI)

\begin{tabular}{|ccccc|}
\hline \multirow{2}{*}{ Phosphorus level } & \multicolumn{5}{c|}{ Time (weeks after sowing) } \\
\cline { 2 - 5 } & 4 & 6 & 8 & 10 \\
\hline \multicolumn{6}{c|}{ First season } \\
\hline 0 P & $0.84 \mathrm{~b}$ & $2.99 \mathrm{~b}$ & $4.11 \mathrm{a}$ & $2.61 \mathrm{a}$ \\
$1 \mathrm{P}$ & $1.25 \mathrm{a}$ & $3.53 \mathrm{a}$ & $4.37 \mathrm{a}$ & $2.67 \mathrm{a}$ \\
$2 \mathrm{P}$ & $1.23 \mathrm{a}$ & $3.58 \mathrm{a}$ & $4.37 \mathrm{a}$ & $2.76 \mathrm{a}$ \\
$3 \mathrm{P}$ & $1.23 \mathrm{a}$ & $3.53 \mathrm{a}$ & $4.41 \mathrm{a}$ & $2.71 \mathrm{a}$ \\
\hline \multicolumn{6}{c}{ Second season } \\
\hline 0 P & $0.74 \mathrm{~b}$ & $1.54 \mathrm{~b}$ & $2.13 \mathrm{~b}$ & $1.28 \mathrm{a}$ \\
\hline $\mathrm{P}$ & $1.03 \mathrm{a}$ & $1.82 \mathrm{a}$ & $2.35 \mathrm{a}$ & $1.28 \mathrm{a}$ \\
$3 \mathrm{P}$ & $1.11 \mathrm{a}$ & $1.91 \mathrm{a}$ & $2.47 \mathrm{a}$ & $1.31 \mathrm{a}$ \\
\hline $\mathrm{P}$ & $1.12 \mathrm{a}$ & $1.93 \mathrm{a}$ & $2.45 \mathrm{a}$ & $1.28 \mathrm{a}$ \\
\hline
\end{tabular}

Means within the same column followed by the same letter are not significantly different at $\mathrm{P} \leq 0.05$ according to Duncan's Multiple Range Test (DMRT). 
Table 5. Effect of nitrogen and phosphorus fertilizers on grain and stover yields

\begin{tabular}{|c|c|c|}
\hline \multirow{2}{*}{ Treatments } & \multicolumn{2}{|c|}{ Yield (ton/ha) } \\
\hline & Grain & Stover \\
\hline & \multicolumn{2}{|c|}{ First season } \\
\hline $0 \mathrm{~N}$ & $1.652 \mathrm{~b}$ & $5.16 \mathrm{c}$ \\
\hline $1 \mathrm{~N}$ & $1.819 \mathrm{~b}$ & $6.92 \mathrm{~b}$ \\
\hline $2 \mathrm{~N}$ & $2.152 \mathrm{a}$ & $7.97 \mathrm{a}$ \\
\hline \multirow[t]{2}{*}{$3 \mathrm{~N}$} & $2.184 \mathrm{a}$ & $8.07 \mathrm{a}$ \\
\hline & \multicolumn{2}{|c|}{ Second season } \\
\hline $0 \mathrm{~N}$ & $0.512 \mathrm{c}$ & $1.6 \mathrm{c}$ \\
\hline $1 \mathrm{~N}$ & $0.653 \mathrm{c}$ & $2.49 \mathrm{~b}$ \\
\hline $2 \mathrm{~N}$ & $0.909 \mathrm{~b}$ & $3.08 \mathrm{ab}$ \\
\hline \multirow[t]{2}{*}{$3 \mathrm{~N}$} & $1.089 \mathrm{a}$ & $3.63 \mathrm{a}$ \\
\hline & \multicolumn{2}{|c|}{ First season } \\
\hline $0 \mathrm{P}$ & $1.781 \mathrm{c}$ & $6.46 \mathrm{~b}$ \\
\hline $1 \mathrm{P}$ & $1.900 \mathrm{bc}$ & $6.90 \mathrm{ab}$ \\
\hline $2 \mathrm{P}$ & $2.018 \mathrm{ab}$ & $7.39 \mathrm{a}$ \\
\hline \multirow[t]{2}{*}{$3 \mathrm{P}$} & $2.109 \mathrm{a}$ & $7.38 \mathrm{a}$ \\
\hline & \multicolumn{2}{|c|}{ Second season } \\
\hline $0 \mathrm{P}$ & $0.668 \mathrm{~b}$ & $2.29 \mathrm{c}$ \\
\hline $1 \mathrm{P}$ & $0.799 \mathrm{a}$ & $2.59 \mathrm{bc}$ \\
\hline $2 \mathrm{P}$ & $0.819 \mathrm{a}$ & $2.82 \mathrm{ab}$ \\
\hline $3 \mathrm{P}$ & $0.857 \mathrm{a}$ & $3.10 \mathrm{a}$ \\
\hline
\end{tabular}

Means within the same column followed by the same letter are not significantly different at $\mathrm{P} \leq 0.05$ according to Duncan's Multiple Range Test (DMRT).

Table 6. Effect of treatment interaction on LAI at the six week after sowing (1st season)

\begin{tabular}{|ccccc|}
\hline \multirow{2}{*}{ Phosphorus level Nitrogen level } \\
\cline { 2 - 5 } & $0 \mathrm{~N}$ & $1 \mathrm{~N}$ & $2 \mathrm{~N}$ & $3 \mathrm{~N}$ \\
\hline $0 \mathrm{P}$ & $1.81 \mathrm{~h}$ & $3.08 \mathrm{efg}$ & $3.68 \mathrm{abcde}$ & $3.38 \mathrm{de}$ \\
$1 \mathrm{P}$ & $3.11 \mathrm{ef}$ & $3.63 \mathrm{abcde}$ & $3.83 \mathrm{abcd}$ & $3.55 \mathrm{bcde}$ \\
$2 \mathrm{P}$ & $2.57 \mathrm{fg}$ & $3.40 \mathrm{de}$ & $4.13 \mathrm{ab}$ & $4.22 \mathrm{a}$ \\
$3 \mathrm{P}$ & $2.48 \mathrm{~g}$ & $3.51 \mathrm{cde}$ & $4.07 \mathrm{abc}$ & $4.07 \mathrm{abc}$ \\
\hline
\end{tabular}

Mean within same column followed by the same letters are not significantly different at $\mathrm{P}<0.05$ according to Duncan's Multiple Range Test (DMRT) 
Table 7. Effect of treatment interaction on plant height $(\mathrm{cm})$ at the six week after sowing ( $2^{\text {nd }}$ season)

\begin{tabular}{|ccccc|}
\hline \multirow{2}{*}{ Phosphorus level } & \multicolumn{4}{c|}{ Nitrogen level } \\
\cline { 2 - 5 } & $0 \mathrm{~N}$ & $1 \mathrm{~N}$ & $2 \mathrm{~N}$ & $3 \mathrm{~N}$ \\
\hline 0 P & $66.07 \mathrm{~h}$ & $79.70 \mathrm{~g}$ & $88.00 \mathrm{f}$ & $99.63 \mathrm{e}$ \\
$1 \mathrm{P}$ & $69.53 \mathrm{~h}$ & $98.70 \mathrm{e}$ & $110.63 \mathrm{~cd}$ & $120.20 \mathrm{ab}$ \\
$2 \mathrm{P}$ & $79.20 \mathrm{~g}$ & $105.67 \mathrm{~cd}$ & $108.23 \mathrm{~cd}$ & $121.43 \mathrm{ab}$ \\
$3 \mathrm{P}$ & $80.67 \mathrm{fg}$ & $104.17 \mathrm{~cd}$ & $115.97 \mathrm{bc}$ & $123.93 \mathrm{a}$ \\
\hline
\end{tabular}

Mean within same column followed by the same letters are not significantly different at $\mathrm{P}<0.05$ according to Duncan's Multiple Range Test (DMRT)

The highest value of LAI was obtained from $3 \mathrm{~N} \times 3 \mathrm{P}$ treatment which the tallest plants were recorded from $3 \mathrm{~N} \times 3 \mathrm{P}$ treatment.

\section{REFERENCES}

Abuelgasim, H.E. and R.P. Jain (1987). Breeding pearl millet for drought in the Sudan. Proceedings of the International Pearl Millet Workshop (7-11 April, 1986). ICRISAT Center India : 307 308.

Batino, A.; C.B. Christianson and A.U. Mokwunye (1989). Soil fertility management of pear millet - producing sandy soil of Sahelian West Africa: The niger experience, soil, crop and water management systems for rainfed agriculture in the Sudano Sahelian zone: Proceedings of an International Workshop (11 - 16 Jan. 1987). ICRISAT Sahelian Center Niamey. Niger: $159-167$.

Batino, A.; C.B. Christianson and W.E. Baethgen (1990). Plant density and ni- trogen fertilizer effects on pearl millet production in Niger. Agron. J. 82: 290 294.

Black, C.A.; D.D. Evans; J.E. Ensminger; J.L. White and F.E. Clark (eds.) (1969). Methods of Soil Analysis. Part 2. Chemical and Microbiological Properties. Madison, Wisconsin, USA.

Elmahi, H.A.; M.E.H. Omer and M.O. Idris, (1995). Dawney mildew of pearl millet in Sudan. International Sorghum and Millet Newsletter (ISMN) No. 36: 50-55.

Elmedani, E.H.Y. (1997). The Effect of Phosphate Rocks, Triple Superphosphate and Their Combinations on the Performance of Sorghum bioclor var. dura L. and Sorghum sudanese (piper) (stapf). pp. 27-35. M.Sc. Thesis Faculty of Agriculture, University of Khartoum.

FAO, (1999). Food and Agricultural Organization of the United Nations, Year Book, No. 53 Rome.

Gomez, K.A. and A.A. Gomez (1984). Statistical Procedures for Agricultural 
Research. $2^{\text {nd }}$ Edition. pp. 75-165. John Wiley and Sons Inc. New York.

Ibrahim, A.E.S. (1996). Growth and forage yield of pearl millet (Pennisetum americanum L.) as affected by irrigation frequency, seed rate and nitrogen in the United Arab Emirates. $U$. of $\boldsymbol{K}$. J. of Agric. Sci. 4(1): 45-60.

Mason, S.C. (1989). Agronomy and cropping systems project $(\mathrm{Nu}-113)$ nitrogen contribution of intercropped cowpea. INTOSORMIL Annual Report: 31- 36. Mourad, S.B.; M.A. El-lakany; A.I. ElAgamy and F.H. Soliman (1986). Response of some varieties of hybrids of maize to plant densities and nitrogen fertilization. I. Growth and flowering traits. Al-Azhar J. Agric. Res. 4: 401- 411.

Okalebo, J.R. (1984). Bulursh millet (Pennisetum typhoides) responses to nitrogen and phosphorus fertilizers at lthookwe, Kitui, Kenya. East Afr. AgricFor. J. 49(3): 62-77.

Singh, K.N. and D.P. Sharma (1995). Effect of subsurface drain spacing and nitrogen level on yield and growth of upland cotton (Gossypium hirsutum) and pearl millet (Pennisetum glaucum) in saline soil. Indian, J. of Agric. Sci. 65 (1):27-33.

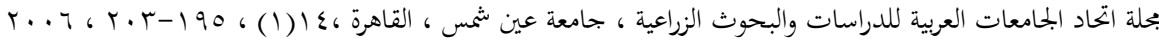 أثز إضافة أسمدة التتروجين والفسفور علي نمو وإنتاجية الدخن المطري في التربة الطينية الطميبة}

$$
\begin{aligned}
& \text { عبد المنعم محمد أحمد التلب' - تاج الاين الثيخ موسي هجو' - }
\end{aligned}
$$

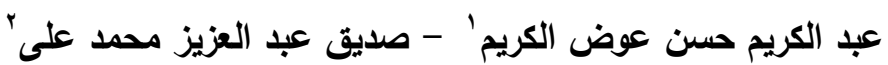

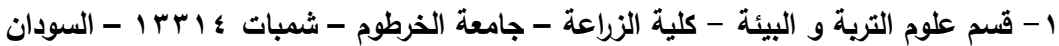

$$
\begin{aligned}
& \text { r - كلية الزراعة - جامعة زالنجـي - ولايـة غرب دارفهور - السـودانه } \\
& \text { أجريت تجربة حقلية لموسمين متتاليين الدخن (درمسا)، وأستخدم أربعـة معامـلات }
\end{aligned}
$$

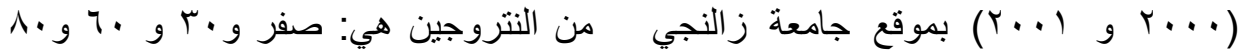

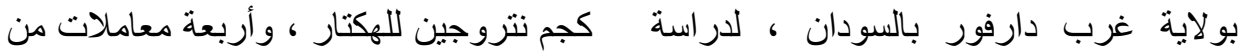

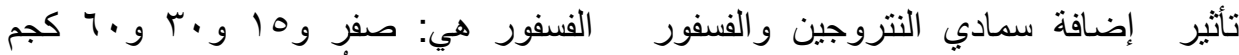

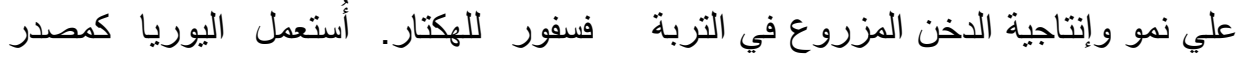

$$
\begin{aligned}
& \text { الطينية الطميية. تم زراعة صنف محلي من للنتروجين (T \% \% و السيوبر فوسفات }
\end{aligned}
$$




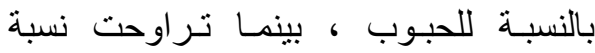

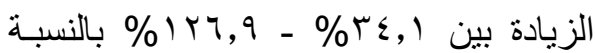
للقصب.

أما الفسفور فقد كان له تأثير معنوي علي طول النبات ودليل مساحة سطح الورقة فئل و إنتاجية الحبوب و القصب ، وقد نراول

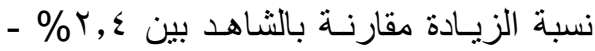

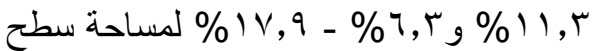

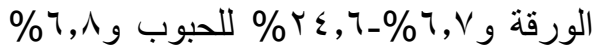

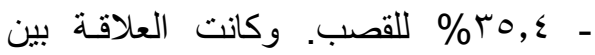

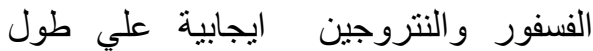
النبات فقط في الاسبوع السادس في الموسم

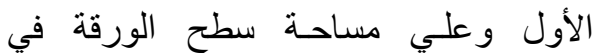

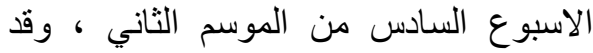

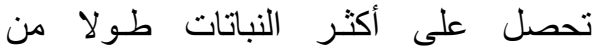
المعاملة r N N N وسجلت أكبر مساحسة

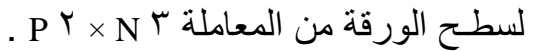

الثلاثي كمصدر للفسفور ( $)$ \% ( وأُتبع في تنفيذ التجربة تصميم القطع المنشقة في خمس مكررات وكانت معاملات النتروجين في القطع الرئيسية ومعاملات

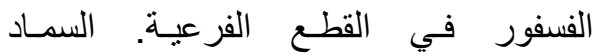
الفسفوري أضيف مع الزر اعة اما النتروجين فقد أضيف علي جرعتين الأولي بعد أربعة الأبة النيف

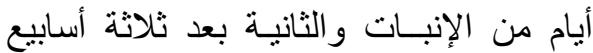

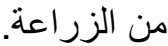
أوضحت النتائج أن إضافـة النتروجين

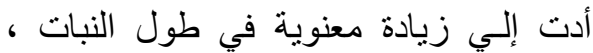

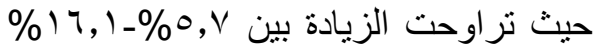

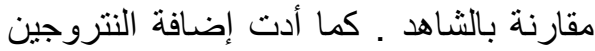

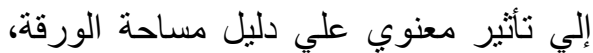

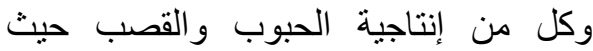

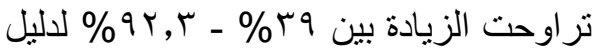

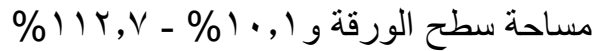

تحيكم: أ.د عبد المنعم محمد الجـلا 